\title{
Measuring lift-off distance and electromagnetic property of metal using dual-frequency linearity feature
}

\author{
Mingyang Lu*, Xiaobai Meng, Ruochen Huang, Liming Chen, Anthony Peyton, Wuliang Yin*
}

\begin{abstract}
Lift-offs of the sensor could significantly affect the measurement signal and reconstruction of material properties when using the electromagnetic (inductive) eddy current sensor. Previously, various methods (including novel sensor designs, and features like zero-crossing frequency, lift-off point of intercept) have been used for eliminating the measurement error caused by the lift-off distance effect of the sensor. However, these approaches can only be applied for a small range of lift-off variations. In this paper, a linear relationship has been found between the sensor liftoff and ratio of dual-frequency eddy current signals, particularly under the high working dual frequencies. Based on this linear relationship, the lift-off variation can be reconstructed firstly with a small error of $2.5 \%$ when its actual value is up to $10 \mathrm{~mm}(10.1$ $\%$ for $20 \mathrm{~mm}$ ). The reconstructed lift-off is used to further get the property of the material under a low single frequency. Experiments on different ferrous metals have been carried out for the testing of the reconstruction scheme. Since the inductance is more sensitive to the material property (and less sensitive to the lift-off) under low frequencies, the reconstruction error of the material property is much smaller than that of the lift-off, with 1.4 $\%$ under $12 \mathrm{~mm}$ (and $4.5 \%$ under $20 \mathrm{~mm}$ ).
\end{abstract}

Index Terms - Eddy current testing; lift-off measurement; property measurement; non-destructive testing; dual-frequency eddy current (DEC) testing.

\section{INTRODUCTION}

$\mathrm{A}$ $\mathrm{S}$ one of the promising and general techniques for nondestructive testing, eddy current (EC) testing has been widely applied for the health checking, flaw inspection, fatigue scanning (e.g. the rolling contact fatigue (RCF) of rail strip), deep exploration, and properties measurement [1-9]. Currently, three types of measurement manner exist, including the multifrequency eddy current (MEC) testing, pulsed eddy current (PEC) testing, and single-frequency eddy current (SEC) testing [10-17]. MEC testing, particularly the swept eddy current testing has its merit of rich information, and high accuracy (better SNR) and commonly applied for the online and off-line measurement. SEC and PEC testing can be used for the online real-time measurement due to its fast feedback response. However, the lift-off of the electromagnetic sensor has been

This work was supported by [UK Engineering and Physical Sciences Research Council (EPSRC)] [grant number: EP/P027237/1] [title: Real-time Inline Microstructural Engineering (RIME)].

M. Lu (Member, IEEE), R. Huang, L. Chen, A. Peyton and W. Yin (Senior Member, IEEE) are with the School of Electrical and Electronic Engineering, University of Manchester, Sackville Street Building, Manchester, M13 9PL, UK (mingyang.lu@manchester.ac.uk; ruochen.huang@manchester.ac.uk; wuliang.yin@manchester.ac.uk) *Corresponding author: M. Lu, W. Yin. found can significantly affect the measured signal, which could further influence the accuracy of inspecting the surface crack and measuring the property (e.g. electrical conductivity, magnetic permeability, and thickness) of materials [18-23].

Various approaches have been proposed to address the liftoff issue on the electromagnetic property measurement of conductive samples, which mainly involves the different sensor designs, measurement techniques and features, and dataprocessing methods. Tian and Sophian have used normalisation and two reference signals to reducing the lift-off effect on PEC testing [20]. Giguère et al have extracted the lift-off point of intersection (LOI) feature from the PEC signals [21]. Wang et al, Angani et al, and Wen et al have used the LOI feature to measure the thickness of coatings and ferromagnetic applications [22-24]. A semi-quadratic system has been designed by Abu-Nabah to reduce the lift-off effect in highfrequency apparent eddy current conductivity spectroscopy [25]. These methods can significantly reduce the lift-off error but only works on a small range of sensor lift-offs.

From our previous work, both the peak frequency feature, zero-crossing feature, and phase feature of the inductance have been proposed for eliminating the lift-off effect on the property measurement of the sample when using both SEC and MEC sensors [26-37]. However, these methods are focusing on reducing the lift-off effect instead of directly getting the lift-off value. Moreover, the proposed techniques are not feasible for a wide range of lift-off variations.

In this paper, a linear relationship, termed as the dualfrequency linearity of lift-off (DLL) feature, has been found between the sensor lift-off and ratio of dual-frequency eddy current (DEC) inductance change (from two sensing pairs). The linearity is more obvious under the high dual frequencies. Based on the linearity, an algorithm has been proposed for the reconstruction of sensor lift-off distance to the teste piece. Experiments on different samples have been carried out. The reconstruction scheme can reconstruct the lift-off distance up to $20 \mathrm{~mm}$. The error of the reconstruction is $2.5 \%$ at $10 \mathrm{~mm}$, and $10.1 \%$ at $20 \mathrm{~mm}$. The reconstructed lift-off is then inputted into

X. Meng is with the Faculty of Art, Science and Technology, University of Northampton, Northampton, NN1 5PH, UK

Author Contributions: (Methodology, manuscript drafting) M. Lu and X. Meng; (conceptualization, manuscript revision) M. Lu; (experiment data curation, manuscript review) M. Lu, R. Huang, L. Chen; (Supervision) W. Yin, A. Peyton, M. Lu. All authors have read and agreed to the published version of the manuscript. 
the iterative inverse solver (modified Newton-Raphson) to further reconstruct the metal property under a low single working frequency ( $2 \mathrm{kHz}$ in this paper). Since the inductance is more (or less) sensitive to the metal property (or lift-off) under low single frequency, the reconstruction error of the metal is much smaller than that of the lift-off, with $1.4 \%$ and $4.5 \%$ under 12 and $20 \mathrm{~mm}$. Moreover, for the measurement of using large sensor lift-offs (e.g. over $14 \mathrm{~mm}$ ), the property reconstruction is even more accurate under slightly lower dualfrequency combinations.

\section{LINEARITY FEATURES AND RECONSTRUCTION ALGORITHMS}

Previously, different features including the zero-crossing feature for the ferromagnetic sample and peak-frequency feature for the non-magnetic sample have been used to eliminate the measurement error caused by the sensor lift-off variations. However, these features are based on the multifrequency mode, which is time-consuming. Besides, the previous scenarios can merely be applied for a small range of lift-offs. Therefore, it is suggested to firstly get the sensor liftoff variation, then accurately reconstruct the metal properties via the interactive inverse solver [28] from the single-frequency measurement data (impedance or inductance).

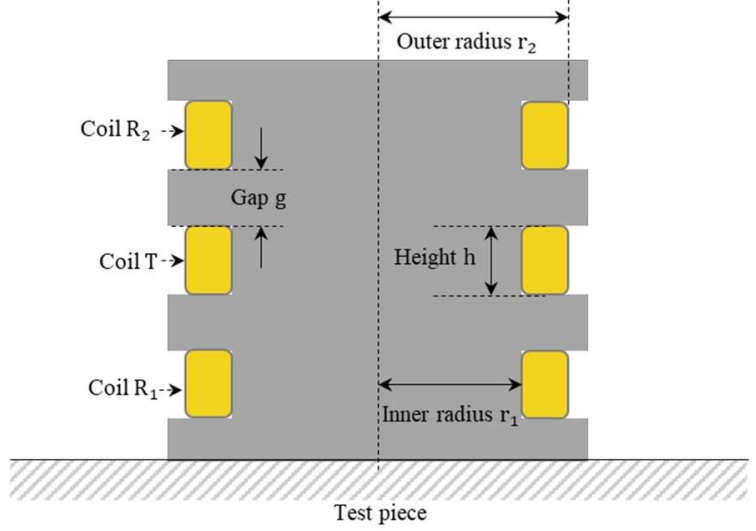

Fig. 1 Air-core sensor above the test piece

\section{A. Analytical formulation}

Though Dodd-Deeds formulation has been proposed over decades, it is still the most frequently cited [4,7,10,26-41] and dominant analytical method for the electromagnetic characteristic of the inductive sensor above both magnetic and non-magnetic conductive materials.

In Fig. 1, according to the Dodd-Deeds formulas for circular coils parallel to the test plate (magnetic materials) [42], the inductance changes exclusive of the background signal (sensor in the air) for both $\mathrm{TR}_{1}$ and $\mathrm{TR}_{2}$ sensing pairs are,

$$
\begin{gathered}
\mathrm{L}_{1}(\omega)=\mathrm{K} \int_{0}^{\infty} \frac{\mathrm{P}^{2}(\alpha)}{\alpha^{6}} \mathrm{e}^{-\alpha\left(2 \mathrm{l}_{0}+\mathrm{g}+\mathrm{h}\right)}\left(\mathrm{e}^{-\alpha \mathrm{h}}-1\right)^{2} \operatorname{Re}(\phi(\alpha)) \mathrm{d} \alpha \\
\mathrm{L}_{2}(\omega)=\mathrm{K} \int_{0}^{\infty} \frac{\mathrm{P}^{2}(\alpha)}{\alpha^{6}} \mathrm{e}^{-\alpha\left(2 \mathrm{l}_{0}+3 \mathrm{~g}+3 \mathrm{~h}\right)}\left(\mathrm{e}^{-\alpha \mathrm{h}}-1\right)^{2} \operatorname{Re}(\phi(\alpha)) \mathrm{d} \alpha
\end{gathered}
$$

In (1) and (2),

$$
\mathrm{K}=\frac{\pi \mu_{0} \mathrm{~N}^{2}\left(\mathrm{r}_{1}+\mathrm{r}_{2}\right)}{2 \mathrm{~h}^{2}\left(\mathrm{r}_{2}-\mathrm{r}_{1}\right)^{2}}
$$

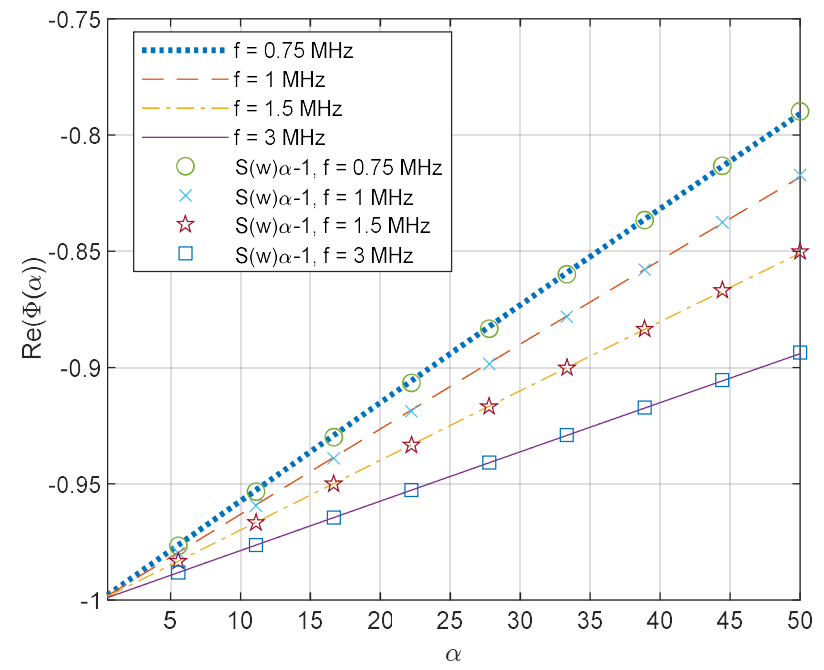

Fig. $2 \operatorname{Re}(\phi(\alpha))$ is linear with $\alpha$ under high frequencies

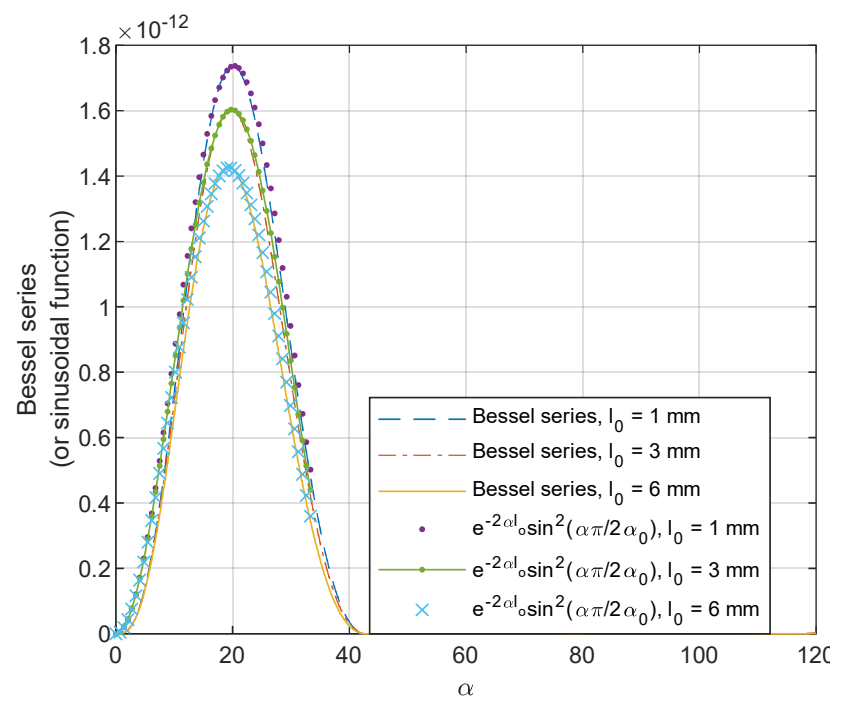

Fig. 3 Bessel series and sinusoidal functions

$r_{1}$ and $r_{2}$ are the inner and outer radii of three identical coils. $h$ is the spiral height of the coil. $\mu_{0}$ is the vacuum magnetic permeability. $\mathrm{N}$ is the number of coil turns. For a general metallic sample (both ferrous and non-magnetic conductive samples), $\phi(\alpha)$ is defined as,

$$
\begin{gathered}
\phi(\alpha)=\frac{\left(\alpha_{1}+\mu_{1} \alpha\right)\left(\alpha_{1}-\mu_{1} \alpha\right)-\left(\alpha_{1}+\mu_{1} \alpha\right)\left(\alpha_{1}-\mu_{1} \alpha\right) \mathrm{e}^{2 \alpha_{1} c}}{-\left(\alpha_{1}-\mu_{1} \alpha\right)\left(\alpha_{1}-\mu_{1} \alpha\right)+\left(\alpha_{1}+\mu_{1} \alpha\right)\left(\alpha_{1}+\mu_{1} \alpha\right) \mathrm{e}^{2 \alpha_{1} c}} \\
\alpha_{1}=\sqrt{\alpha^{2}+j \omega \sigma \mu_{1} \mu_{0}} \\
\mathrm{P}(\alpha)=\int_{\alpha \mathrm{r}_{1}}^{\alpha \mathrm{r}_{2}} \rho J_{1}(\rho) \mathrm{d} \rho
\end{gathered}
$$

In (5), $\omega$ is the working angular frequency for the excitation current flowing in the coil. $\mu_{1}$ and $\sigma$ are the relative magnetic permeability and electrical conductivity of the sample. In (6), $J_{1}$ is the first-order Bessel function of the first kind.

\section{B. Linearity feature}

The phase term $\operatorname{Re}(\phi(\alpha))$ in (1) and (2) is found to be linear to the integral variable $\alpha$ under high frequencies. As Fig. 2 depicts, the slope of the line is denoted as $S(\omega)$. The relation between $S(\omega)$ and $\operatorname{Re}(\phi(\alpha))$ is, 


$$
\operatorname{Re}(\phi(\alpha))=S(\omega) \alpha-1
$$

$S(\omega)$ in (7) has been found related to the sample properties and working (angular) frequency $\omega$. Besides, the slope $-S(\omega)$ is proportional to $1 / \sqrt{\omega}$.

Substitute (7) into (1) and (2), The inductance from $\mathrm{TR}_{1}$ and $\mathrm{TR}_{2}$ becomes,

$$
\begin{gathered}
\mathrm{L}_{1}(\omega)=\mathrm{K} \int_{0}^{\infty} \frac{\mathrm{P}(\alpha)}{\alpha^{6}} \mathrm{e}^{-\alpha\left(2 \mathrm{l}_{0}+\mathrm{g}+\mathrm{h}\right)}\left(\mathrm{e}^{-\alpha \mathrm{h}}-1\right)^{2}(\mathrm{~S}(\omega) \alpha-1) \mathrm{d} \alpha \\
\mathrm{L}_{2}(\omega)=\mathrm{K} \int_{0}^{\infty} \frac{\mathrm{P}(\alpha)}{\alpha^{6}} \mathrm{e}^{-\alpha\left(2 \mathrm{l}_{0}+3 \mathrm{~g}+3 \mathrm{~h}\right)}\left(\mathrm{e}^{-\alpha \mathrm{h}}-1\right)^{2}(\mathrm{~S}(\omega) \alpha-1) \mathrm{d} \alpha
\end{gathered}
$$

As Fig. 3 illustrates, the Bessel series $\frac{P_{1}(\alpha)}{\alpha^{5}} e^{-\alpha\left(2 l_{0}+g+h\right)}$ $\left(\mathrm{e}^{-2 \alpha \mathrm{h}}-2 \mathrm{e}^{-\alpha \mathrm{h}}+1\right)$ can be approximated as a product of a sinusoidal function $\sin ^{2}\left(\frac{\alpha \pi}{2 \alpha_{0}}\right)$ peaking at $\alpha_{0}$ and exponential term $\mathrm{e}^{-2 \alpha \mathrm{l}_{0}}$. Thus, in (8) and (9),

$$
\begin{gathered}
\mathrm{S}(\omega) \int_{0}^{\infty} \frac{\mathrm{P}(\alpha)}{\alpha^{5}} \mathrm{e}^{-\alpha\left(2 \mathrm{l}_{0}+\mathrm{g}+\mathrm{h}\right)}\left(\mathrm{e}^{-\alpha \mathrm{h}}-1\right)^{2} \mathrm{~d} \alpha \\
=\mathrm{BS}(\omega) \int_{0}^{2 \alpha_{0}} \mathrm{e}^{-2 \alpha \mathrm{l}_{0}} \sin ^{2}\left(\frac{\alpha \pi}{2 \alpha_{0}}\right) \mathrm{d} \alpha \\
=\frac{\pi^{2} \mathrm{BS}(\omega)\left(1-\mathrm{e}^{-4 \alpha_{0} \mathrm{l}_{0}}\right)}{4 \mathrm{l}_{0}\left(4 \alpha_{0}^{2} \mathrm{l}_{0}^{2}+\pi^{2}\right)} \\
\mathrm{S}(\omega) \int_{0}^{\infty} \frac{\mathrm{P}(\alpha)}{\alpha^{5}} \mathrm{e}^{-\alpha\left(2 \mathrm{l}_{0}+3 \mathrm{~g}+3 \mathrm{~h}\right)}\left(\mathrm{e}^{-\alpha \mathrm{h}}-1\right)^{2} \mathrm{~d} \alpha \\
=\mathrm{BS}(\omega) \int_{0}^{2 \alpha_{0}} \mathrm{e}^{-2 \alpha\left(\mathrm{l}_{0}+\mathrm{g}+\mathrm{h}\right)} \sin ^{2}\left(\frac{\alpha \pi}{2 \alpha_{0}}\right) \mathrm{d} \alpha \\
=\frac{\pi^{2} \mathrm{BS}(\omega)\left(1-\mathrm{e}^{-4 \alpha_{0}\left(\mathrm{l}_{0}+\mathrm{g}+\mathrm{h}\right)}\right)}{4 \mathrm{l}_{0}\left(4 \alpha_{0}^{2}\left(\mathrm{l}_{0}+\mathrm{g}+\mathrm{h}\right)^{2}+\pi^{2}\right)}
\end{gathered}
$$

In (10) and (11), $\alpha_{0}$, termed as the spatial frequency, is determined by the geometry of the sensor (proportional to the reciprocal of sensor radius). $\mathrm{B}$ is the normalization factor between the Bessel terms and sinusoidal function.

$$
\mathrm{B}=\frac{\mathrm{P}\left(\alpha_{0}\right)}{\alpha_{0}^{5}} \mathrm{e}^{-\alpha_{0}(g+h)}\left(\mathrm{e}^{-\alpha_{0} \mathrm{~h}}-1\right)^{2}
$$

Substitute (10) and (11) into (8) and (9), $\mathrm{L}_{1}(\omega)$ and $\mathrm{L}_{2}(\omega)$ become,

$$
\begin{gathered}
\mathrm{L}_{1}(\omega)=\mathrm{K}\left(\frac{\pi^{2} \mathrm{BS}(\omega)\left(1-\mathrm{e}^{-4 \alpha_{0} \mathrm{l}_{0}}\right)}{4 \mathrm{l}_{0}\left(4 \alpha_{0}^{2} \mathrm{l}_{0}^{2}+\pi^{2}\right)}-\mathrm{H}_{1}\right) \\
\mathrm{L}_{2}(\omega)=\mathrm{K}\left(\frac{\pi^{2} \mathrm{BS}(\omega)\left(1-\mathrm{e}^{-4 \alpha_{0}\left(\mathrm{l}_{0}+\mathrm{g}+\mathrm{h}\right)}\right)}{4\left(\mathrm{l}_{0}+\mathrm{g}+\mathrm{h}\right)\left(4 \alpha_{0}^{2}\left(\mathrm{l}_{0}+\mathrm{g}+\mathrm{h}\right)^{2}+\pi^{2}\right)}-\mathrm{H}_{2}\right)
\end{gathered}
$$

Where in (13) and (14), $\mathrm{H}_{1}$ and $\mathrm{H}_{2}$ are,

$$
\begin{aligned}
\mathrm{H}_{1} & =\int_{0}^{\infty} \frac{\mathrm{P}(\alpha)}{\alpha^{6}} \mathrm{e}^{-\alpha\left(2 \mathrm{l}_{0}+\mathrm{g}+\mathrm{h}\right)}\left(\mathrm{e}^{-\alpha \mathrm{h}}-1\right)^{2} \mathrm{~d} \alpha \\
\mathrm{H}_{2} & =\int_{0}^{\infty} \frac{\mathrm{P}(\alpha)}{\alpha^{6}} \mathrm{e}^{-\alpha\left(2 \mathrm{l}_{0}+3 \mathrm{~g}+3 \mathrm{~h}\right)}\left(\mathrm{e}^{-\alpha \mathrm{h}}-1\right)^{2} \mathrm{~d} \alpha
\end{aligned}
$$

The exponential term in (13) can be estimated using the Padé approximation. Besides, $4 \alpha_{0}^{2} l_{0}^{2} \ll \pi^{2}$ for the large coil. Therefore, (13) becomes

$$
\mathrm{L}_{1}(\omega)=\mathrm{K}\left(\frac{\mathrm{BS}(\omega) \alpha_{0}}{0.72 \alpha_{0} \mathrm{l}_{0}+1}-\mathrm{H}_{1}\right)
$$

The value 0.72 in (17) is determined by the expansion point of zero when using the Padé approximation.

In (14), since $\mathrm{l}_{0} \ll(\mathrm{g}+\mathrm{h})$ satisfies for a coil with large spiral height $h$ and gap g, (14) can be approximated as,

$$
L_{2}(\omega)=K\left(\frac{\pi^{2} B S(\omega)\left(1-e^{-4 \alpha_{0}(g+h)}\right)}{4(g+h)\left(4 \alpha_{0}^{2}(g+h)^{2}+\pi^{2}\right)}-H_{2}\right)
$$

Assume the measurement is operated under dual high (angular) frequency $\omega_{1}$, and $\omega_{2}$. Then, the inductance difference using both $\mathrm{TR}_{1}$ and $\mathrm{TR}_{2}$ are,

$$
\begin{aligned}
& \Delta \mathrm{L}_{1}= \mathrm{L}_{1}\left(\omega_{1}\right)-\mathrm{L}_{1}\left(\omega_{2}\right)=\frac{\mathrm{KB}_{0}\left(\mathrm{~S}\left(\omega_{2}\right)-\mathrm{S}\left(\omega_{1}\right)\right)}{0.72 \alpha_{0} \mathrm{l}_{0}+1} \\
& \Delta \mathrm{L}_{2}=\mathrm{L}_{2}\left(\omega_{1}\right)-\mathrm{L}_{2}\left(\omega_{2}\right) \\
&=\frac{\pi^{2} \mathrm{~KB}\left(\mathrm{~S}\left(\omega_{2}\right)-\mathrm{S}\left(\omega_{1}\right)\right)\left(1-\mathrm{e}^{-4 \alpha_{0}(\mathrm{~g}+\mathrm{h})}\right)}{4(\mathrm{~g}+\mathrm{h})\left(4 \alpha_{0}^{2}(\mathrm{~g}+\mathrm{h})^{2}+\pi^{2}\right)}
\end{aligned}
$$

Combine (19) and (20), the lift-off of the sensor can be derived from the inductance of dual high frequency.

$$
\mathrm{l}_{0}=\frac{5.56\left(4 \alpha_{0}^{2}(\mathrm{~g}+\mathrm{h})^{2}+\pi^{2}\right)(\mathrm{g}+\mathrm{h}) \Delta \mathrm{L}_{2}}{\pi^{2}\left(1-\mathrm{e}^{-4 \alpha_{0}(\mathrm{~g}+\mathrm{h})}\right) \Delta \mathrm{L}_{1}}-\frac{1.39}{\alpha_{0}}
$$

It can be found the lift-off of the sensor is linear to the highfrequency inductance ratio $\Delta \mathrm{L}_{2} / \Delta \mathrm{L}_{1}$ of two sensing pairs $\left(\mathrm{TR}_{1}\right.$ and $\left.\mathrm{TR}_{2}\right)$.

After getting the lift-off of the sensor, the properties of the slab - either the electrical conductivity $\sigma$ or magnetic permeability $\mu_{1}$ could be reconstructed by inputting the derived lift-off into equation (1) (The thickness of thin metallic plates can also be reconstructed if the skin depth is larger than the actual thickness). The solution of the property ( $\mu_{1}$ or $\sigma$ ) can be found using the inverse solver - Newton-Raphson iterative method [26]. The principle of the inverse solver is finding the local minimum of the difference between the measured inductance and analytical value (equation 1) when varying (decreasing or increasing, depending on the Jacobian value) the reference value of the unknown property.

\section{EXPERIMENT SETUP}

Experiments on different samples have been carried out for the performance testing of the proposed lift-off algorithm equation (21). The inductance measurement for the sensor above the test piece is under the multi-frequency mode (ranges from 10 to $3 \mathrm{MHz}$ ) to test the accuracy of the reconstruction under different dual-frequency combinations. By subtracting the reference signals when the sensor is deployed in the free space, the ambient noise signals (including the skin effect and proximity of coil windings) can be significantly eliminated, particularly under high working frequencies. This calibration method has been used and verified in previous articles [26-39].

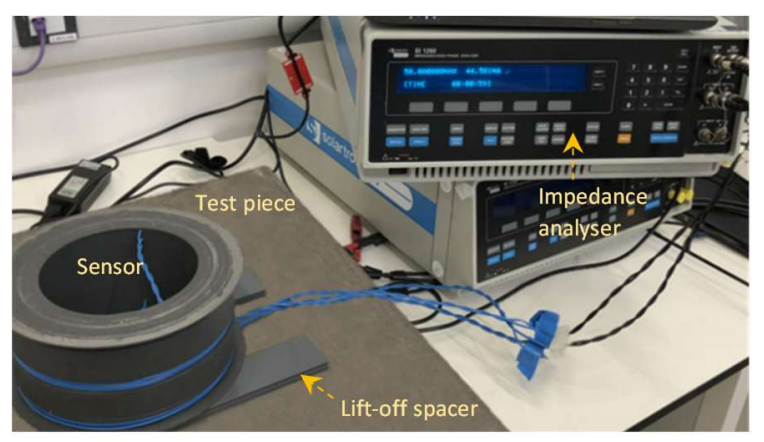

Fig. 4 Air-core sensor connected to the impedance analyser (SL1260, Solartron). 
Due to the air-core setup and limited thickness of the wire, the magnetic field generated by the exciting current is not considerable compared to that of the ferromagnetic core sensor. Therefore, the air-core sensor is commonly used for the measurement of the initial permeability of the magnetic material (instead of the data under remanence magnetization or residual magnetism). Considering the analytical inversion of the data and potential of the high-temperature environment (the ferrous silicon steel core will melt), the sensor is selected as the air-core setup. As Fig. 4 illuminates, the air-core sensor is connected to the impedance analyser. The sensor is deployed above the sample with a series of lift-offs using the lift-off spacers. The sensor is composed of three equally separated spiral coils with identical parameters listed in Table 1 . Since the Bessel function $P(\alpha)$ will shift left-side as $r_{1}$ increases, a sinusoidal function peaking at a larger spatial frequency $\alpha_{0}$ is needed for the approximation in Fig. 3. Besides, from the previous articles, it has been found the spatial frequency $\alpha_{0}$ is proportional to the reciprocal of the inner radius of the sensor [43]. Therefore, the sensor is designed with a large inner radius $(89.0 \mathrm{~mm})$, to ensure the spatial frequency $\alpha_{0}$ is small enough (22.77 for the sensor dimension in Table 1) for the approximation condition $4 \alpha_{0}^{2} l_{0}^{2} \ll \pi^{2}$ used in (13) and (14) $\left(4 \alpha_{0}^{2} 1_{0}^{2} \approx 0.83\right.$ for the lift-off of $20.0 \mathrm{~mm}$ ). Consequently, a small spatial frequency $\alpha_{0}$ allows an extensive range of lift-off variations.

TABLE I

SENSOR PARAMETERS

\begin{tabular}{|c|c|}
\hline Coil Parameters & Value \\
\hline Inner radius $-r_{1}(\mathrm{~mm})$ & 89.0 \\
\hline Outer radius $-r_{2}(\mathrm{~mm})$ & 90.0 \\
\hline Turns - N & 10 \\
\hline Spiral height - $\mathrm{h}(\mathrm{mm})$ & 10.0 \\
\hline Gap - g (mm) & 35.0 \\
\hline Lift-offs - $\mathrm{l}_{0}(\mathrm{~mm})$ & $1.0: 1.0: 20.0$ \\
\hline Spatial frequency $-\alpha_{0}$ & 22.77 \\
\hline
\end{tabular}

As listed in Table 2, samples are selected as two ferromagnetic plates - the (ferrite-austenite) dual-phase (DP) 600 and high-tensile Cr-Mo steels. The selection of different materials is to test whether and how the lift-off algorithm is independent of the metal properties. The reconstructed sensor lift-off is further used to measure the relative magnetic permeability and electrical conductivity of DP-600 and Cr-Mo steels via the inverse solver.

TABLE II

PROPERTIES OF FERROMAGNETIC MATERIALS

\begin{tabular}{ccc}
\hline \hline Properties & DP 600 & $\begin{array}{c}\text { Cr-Mo steel } \\
\text { (High-tensile) }\end{array}$ \\
\hline $\begin{array}{c}\text { Thickness (mm) } \\
\text { Electrical conductivity } \\
(\mathrm{MS} / \mathrm{m})\end{array}$ & 5.4 & 6.0 \\
$\begin{array}{c}\text { Relative magnetic } \\
\text { permeability }\end{array}$ & 4.13 & 3.37 \\
\hline \hline
\end{tabular}

\section{RESUlts}

\section{A. Multi-frequency inductance}

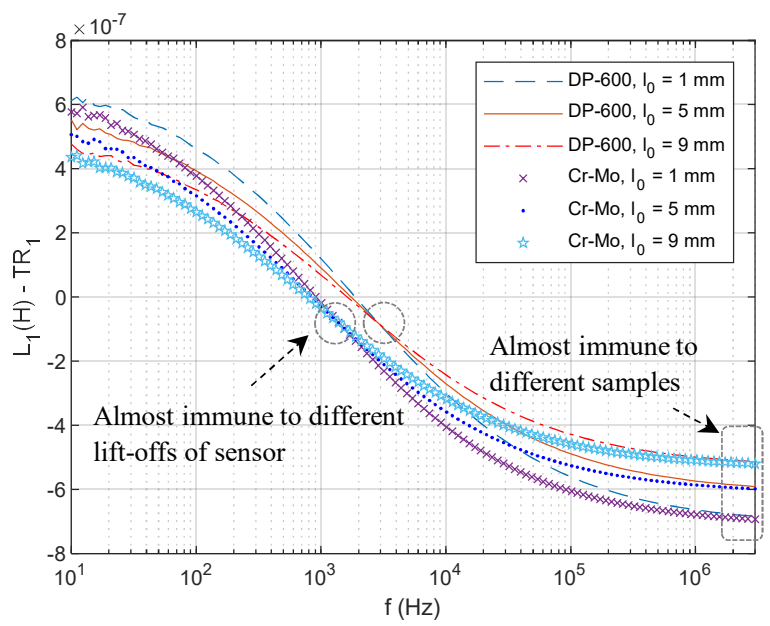

(a)

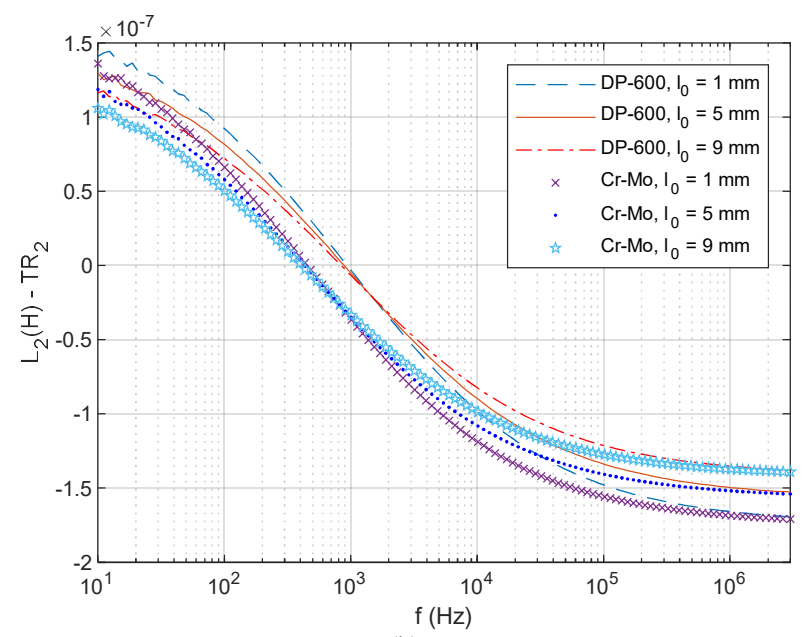

(b)

Fig. 5 Multi-frequency inductance data for the sensor above the DP-600 and Cr-Mo steel under the lift-off of 1,5 , and $9 \mathrm{~mm}$ (a) $\mathrm{TR}_{1}$ sensing pair (b) $\mathrm{TR}_{2}$ sensing pair

Fig. 5 exhibits the measured multi-frequency inductance using $\mathrm{TR}_{1}$ and $\mathrm{TR}_{2}$ sensing pairs when the sensor is deployed above the sample (DP-600 and Cr-Mo steel) with a lift-off distance of 1,5 , and $9 \mathrm{~mm}$. The fluctuating noise signal exists under the lower-frequency spectrum (10 to $1 \mathrm{kHz}$ ) due to the low Signal Noise Ratio (SNR) of the instrument. Moreover, a very higher frequency will result in distortion due to the resonance effect of the impedance analyser. Since $\mathrm{R}_{1}$ coil is closer to the test piece (as Fig. 1 depicts), the magnitude of its inductance data is much larger than that from $\mathrm{R}_{2}$ coil. It can be observed that the inductance curves cross zero around $1 \mathrm{kHz}$, which is termed as the zero-crossing frequency. Previously, the multi-frequency feature - zero-crossing frequency is used to reconstruct the magnetic permeability of the sample. However, as Fig. 5 demonstrates, the zero-crossing frequency is less affected but still influenced by the sensor lift-off. Besides, it can be seen from Fig. 5 that inductance curves for the sample with different sensor lift-offs overlap under a specific frequency (slightly larger than the zero-crossing frequency). The 
corresponded frequency at the overlapped point (dash circular in Fig. 5 (a)) is the optimal one for the inverse of the metal properties due to its immunity to the sensor lift-off. However, it is tricky to get the optimal working frequency (at the overlapped point) for different samples. Therefore, it is recommended to reconstruct the exact value of the sensor liftoff and use it to accurately inverse the metal properties via the inverse solver.

As can be observed from the dashed block area in Fig. 5 (a), the inductance curves are found to be gradually more sensitive to the sensor lift-off and immune to the test piece (as the inductance curve for different samples with the same sensor liftoff gradually overlaps) under the high frequencies. Thus, it is suggested to reconstruct the lift-off information from the highfrequency inductance data.

Since the high-frequency inductance is almost independent of the sample properties, for a certain unknown sensor lift-off, it is a reasonable hypothesis that the lift-off distance of the sensor can be inversed from the information of two sensing pairs $\left(\mathrm{TR}_{1}\right.$ and $\left.\mathrm{TR}_{2}\right)$.

B. Relation between ratio - $\Delta L_{2} / \Delta L_{1}$ and sensor lift-off for the whole working frequency range

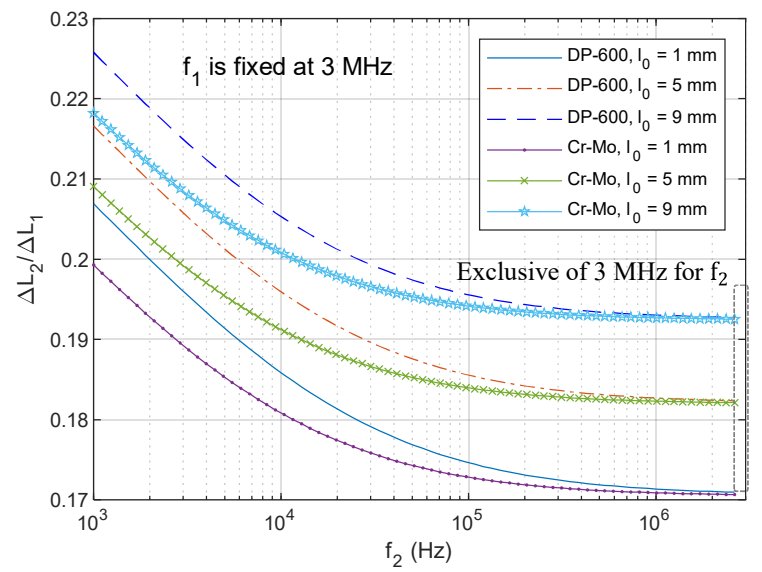

(a)

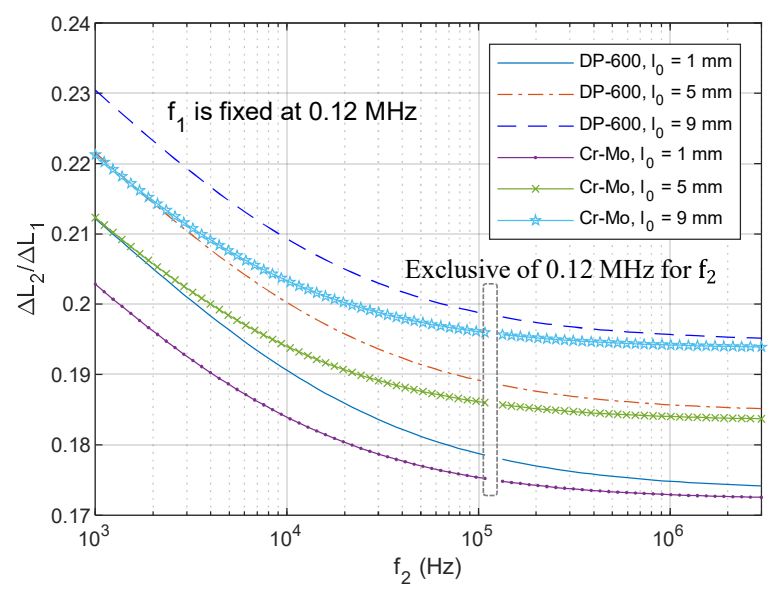

(b)

Fig. 6 Ratio of the dual-frequency inductance difference $-\Delta \mathrm{L}_{2} / \Delta \mathrm{L}_{1}$ under different $\mathrm{f}_{2}$, with the other frequency $\mathrm{f}_{1}$ fixed at (a) $3 \mathrm{MHz}$ (b) $0.12 \mathrm{MHz}$

It has been found the ratio between the slope of the highfrequency inductance-frequency curve from $\mathrm{TR}_{1}$ and $\mathrm{TR}_{2}$ is

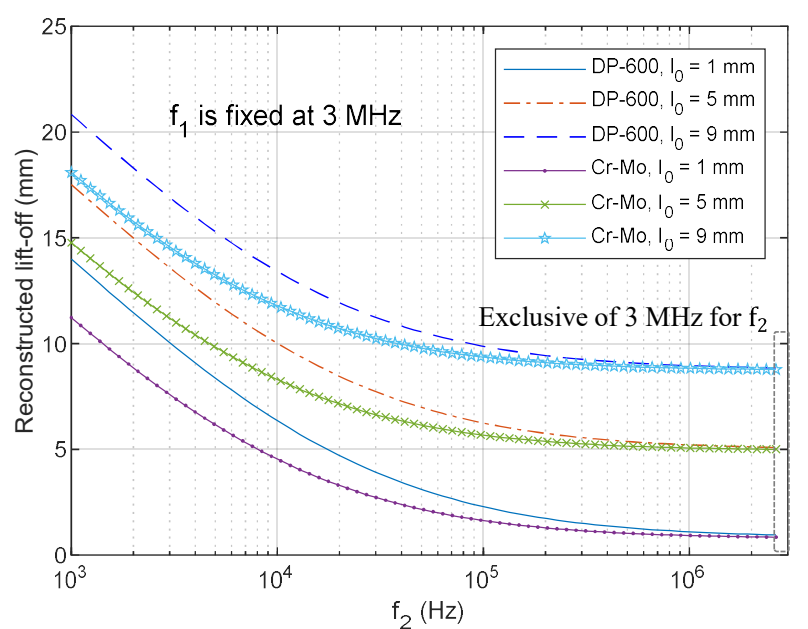

(a)

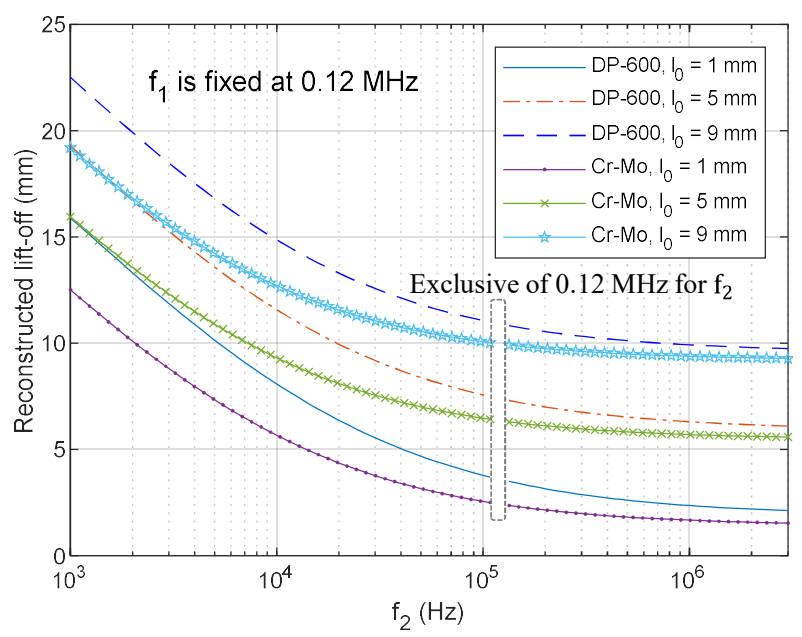

(b)

Fig. 7 Reconstructed sensor lift-off under different $f_{2}$, with the other frequency $\mathrm{f}_{1}$ fixed at (a) $3 \mathrm{MHz}$ (b) $120 \mathrm{kHz}$

linear to the sensor lift-off. As shown in Fig. 6, the different of the inductance $\left(\Delta \mathrm{L}_{1}\right.$ and $\left.\Delta \mathrm{L}_{2}\right)$ is selected under dual hight frequencies, with one frequency $\mathrm{f}_{1}$ fixed (at 0.12 and $3 \mathrm{MHz}$ ) and the other $\mathrm{f}_{2}$ varying from $1 \mathrm{kHz}$ to $3 \mathrm{MHz}$ (exclusive of $\mathrm{f}_{1}$ 0.12 and $3 \mathrm{MHz}$ ). It can be observed the ratio $\Delta \mathrm{L}_{2} / \Delta \mathrm{L}_{1}$ for different samples decreases, gradually becomes (almost but would not absolutely) stable, and overlaps (under the same sensor lift-off) with increased $\mathrm{f}_{2}$. Compared with Fig. 6 (b) ( $\mathrm{f}_{1}$ is fixed at $0.12 \mathrm{MHz}$ ), the high-frequency inductance in Fig 6 (a) ( $f_{1}$ is fixed at a higher frequency - $3 \mathrm{MHz}$ ) is more independent of different test pieces. Moreover, the highfrequency value of ratio $\Delta \mathrm{L}_{2} / \Delta \mathrm{L}_{1}$ is found to be linear to the increased sensor lift-offs. (With a step of $4 \mathrm{~mm}$ for the increased lift-off from 1 to $9 \mathrm{~mm}$, gaps between three overlapped curves are nearly identical under the high frequency.)

Since the ratio $\Delta \mathrm{L}_{2} / \Delta \mathrm{L}_{1}$ is found to be linear with the sensor lift-offs, the lift-off can be reconstructed via the proposed formulation - equation (21). As (21) depicts, the linear correlation factor between the ratio $\Delta \mathrm{L}_{2} / \Delta \mathrm{L}_{1}$ and lift-off distance is determined by the geometric information of the sensor, including the coil height $\mathrm{h}$, coil gap g, and spatial frequency $\alpha_{0}$ (related to the radius of sensor coil). In Fig. 7, by using the coil parameters listed in Table 1, the lift-off of the 
sensor can be inversed from the ratio $\Delta \mathrm{L}_{2} / \Delta \mathrm{L}_{1}$ in Fig. 6 . One of the dual frequencies $\mathrm{f}_{1}$ is fixed at both 0.12 and $3 \mathrm{MHz}$. The other frequency $\mathrm{f}_{2}$ ranges from $1 \mathrm{kHz}$ to $3 \mathrm{MHz}$ but exclusive of the value of frequency $f_{1}\left(\Delta \mathrm{L}_{1}\right.$ and $\Delta \mathrm{L}_{2}$ will be zero if dual frequencies are identical). Similar to the trend in Fig. 6, the reconstructed sensor lift-off achieves its best performance under the high frequency. Under $1 \mathrm{kHz}$, the reconstructed liftoff is much larger than the actual values and mainly dependent on the test piece (and almost immune to the actual value). Besides, the reconstructed sensor lift-off behaves more independent of different samples when the dual frequencies $\left(\mathrm{f}_{1}\right.$ and $f_{2}$ ) are both high enough. As depicted in Fig. 7 (a), the liftoff of the sensor can be accurately inversed via (21) with a small error of $4 \%$.

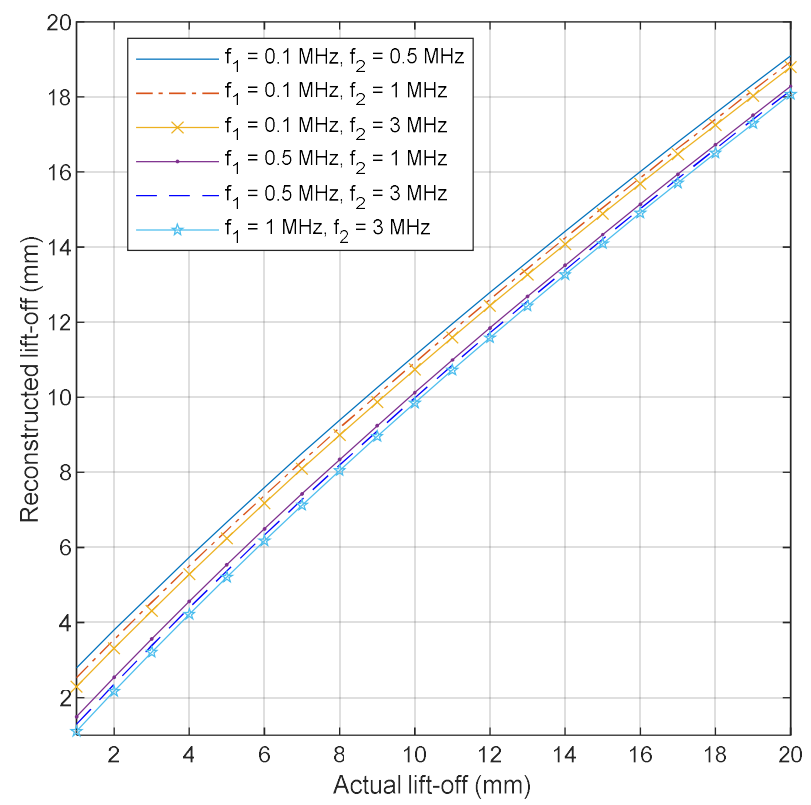

(a)

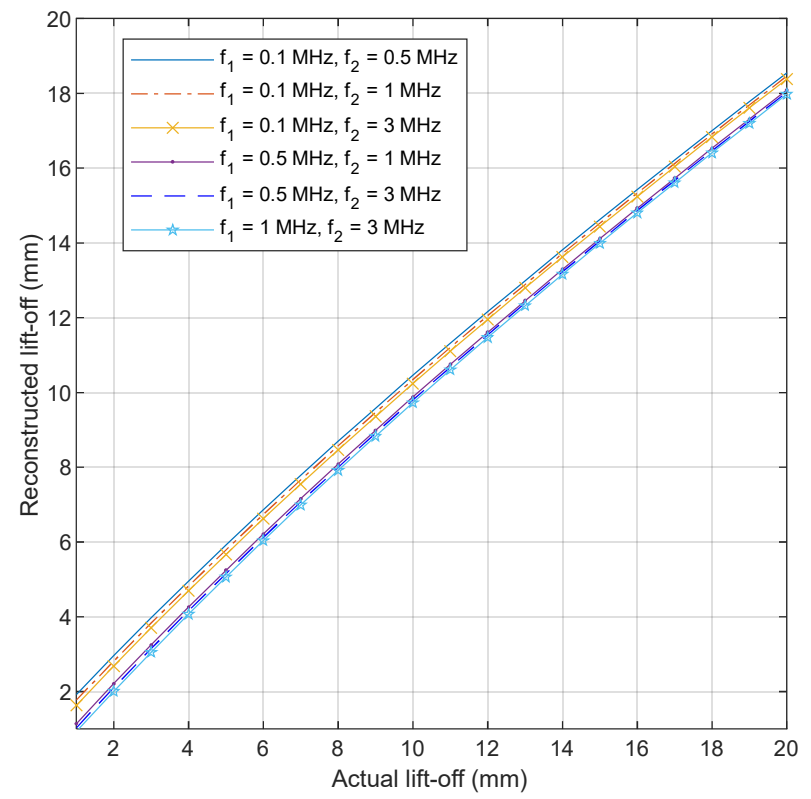

(b)

Fig. 8 Reconstructed sensor lift-off under different pairings of the dual frequencies $f_{1}$ and $f_{2}$ when the sensor is above the (a) DP-600 steel (b) Cr-Mo steel

\section{Reconstruction of sensor lift-off (wide range) under high working frequencies}

As the lift-off distance between sensor and sample can be reconstructed accurately with a small range of actual lift-offs (from 1 to $9 \mathrm{~mm}$ ), it is necessary to further investigate the performance of the reconstruction scheme (equation 21) under a wide range of sensor lift-offs. Fig. 8 denotes the reconstructed value when the sensor is lifted to a maximum distance of $20 \mathrm{~mm}$ to the test piece. To investigate the reconstruction scenario under different combinations of frequency (both different magnitudes and frequency gaps - $\left(f_{2}-f_{1}\right)$ ), the dual high frequencies are randomly selected from $0.1,0.5,1$, and $3 \mathrm{MHz}$. From Fig. 8, lower dual frequencies combinations will result in an over large reconstructed lift-off when its actual value (liftoff) is less than $16 \mathrm{~mm}$. Moreover, the reconstructed value varies with different samples when using low dual frequencies combinations (with the reconstructed lift-off of $\mathrm{Cr}-\mathrm{Mo}$ case more accurate than that of DP-600 steel). Overall, the reconstruction of sensor lift-off achieves better performance when both dual frequencies $\left(\mathrm{f}_{1}\right.$ and $\mathrm{f}_{2}$ ) are high. For example, when the dual frequencies are selected as $1 \mathrm{MHz}$ and $3 \mathrm{MHz}$, the reconstructed curve is almost the same for both samples (independent of the test piece). Besides, the maximum error of the lift-off reconstruction is around $2.5 \%$ within $10 \mathrm{~mm}$, and $10.1 \%$ within $20 \mathrm{~mm}$.

\section{Reconstruction of metal property}

The reconstructed lift-off can be inputted into equation (1) to further inverse the electromagnetic property of the test piece. By using the inverse solver - Newton-Raphson iterative method [26], properties of the sample including the relative magnetic permeability of DP-600 steel and electrical conductivity of CrMo steel (Table 2) can be accurately reconstructed. The principle of the inverse solver is exploiting the local minimum of the difference between the measured inductance and analytical value (equation 1) when fitting/amending (either decreasing or increasing, based on the Jacobian value) the value of the unknown property. Since the inductance is more (or less) sensitive to the metal property (or lift-off) under low frequencies (as shown in Fig. 5), a low frequency of $2 \mathrm{kHz}$ and

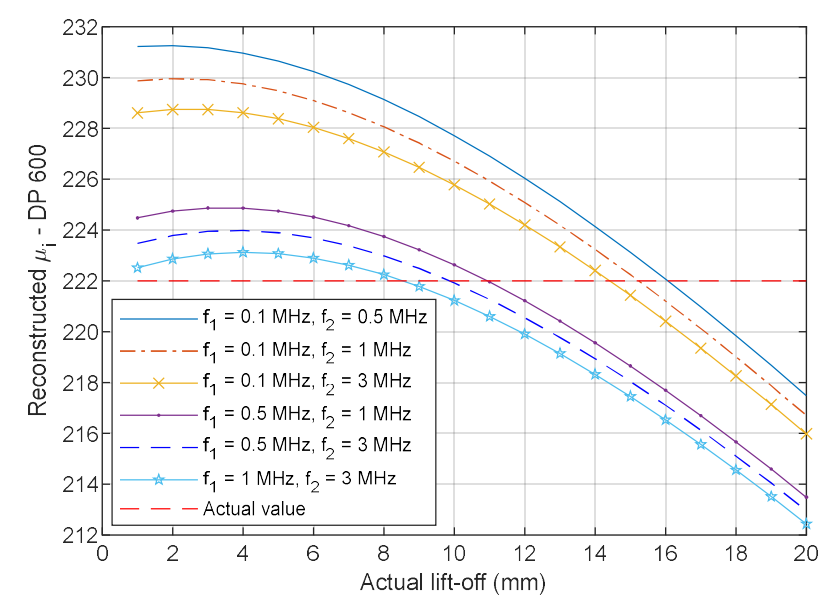

(a) 


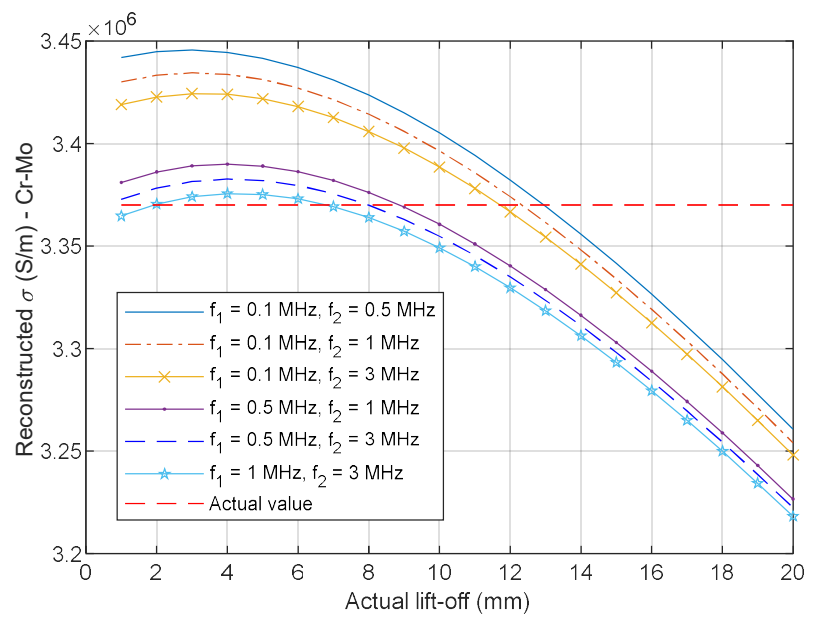

(b)

Fig. 9 Measured metal properties under $2 \mathrm{kHz}$ from the reconstructed liftoff using different pairings of the dual frequencies $\mathrm{f}_{1}$ and $\mathrm{f}_{2}$ (a) Relative magnetic permeability of DP-600 steel (b) Electrical conductivity of CrMo steel

its corresponding inductance are used for the inversion of metal property. Consequently, the reconstruction error of the metal properties is much smaller than that of the sensor lift-off (which is $10.1 \%$ on $20 \mathrm{~mm}$ ). As illustrated in Fig. 9, by using the combination of large dual frequencies ( 1 and $3 \mathrm{MHz}$ ), the relative magnetic permeability of DP-600 (or electrical conductivity of $\mathrm{Cr}-\mathrm{Mo}$ ) can be reconstructed with a very small error of less than $1 \%$ (or $1.4 \%$ ) when the lift-off is within 12 $\mathrm{mm}$, and $4.5 \%$ (or $4.4 \%$ ) when the lift-off is $20 \mathrm{~mm}$. Besides, it can be observed from both (a) and (b) of Fig. 9 that, the lowest dual-frequency combination $(0.1$ and $0.5 \mathrm{MHz})$ even has a better performance when the lift-off of the sensor is larger than $14 \mathrm{~mm}$, which provides an optional selection of the dual frequencies for the measurement with large sensor lift-offs.

\section{CONCLUSIONS}

A dual-frequency linearity of the lift-off (DFL) feature has been found in this paper. That is, the lift-off of the sensor is found to be linear with the ratio of dual-frequency inductance change from two sensing pairs. The feature is more obvious under high working dual frequencies. Based on the DFL feature, an algorithm has been proposed for the reconstruction of the sensor lift-off. Experiments on different ferromagnetic samples have been carried out. The proposed lift-off reconstruction scheme is found can directly get the lift-off value from the dual-frequency inductance of two sensing pairs. The inductance for the eddy current sensor above the sample has been found to be almost immune to the test piece under high dual frequencies. Consequently, the reconstructed lift-off is found independent of the test piece under high dual frequencies. From the experiments, the lift-off can be reconstructed with an error of $2.5 \%$ under $10 \mathrm{~mm}$, and $10.1 \%$ under $20 \mathrm{~mm}$. The reconstructed lift-off is then inputted into the inverse solver for the further measurement of metal properties. Since the inductance is more sensitive to the materials, and less sensitive to the sensor lift-offs at lower frequencies (overlapped point for one sample under different lift-offs in Fig. 5; slightly larger than the zero-crossing frequency), the single working frequency for the inversion of metal properties is selected at $2 \mathrm{kHz}$. Therefore, reconstructed metal properties even have a smaller error of 1.4 $\%$ and $4.5 \%$ under the sensor lift-off of 12 and $20 \mathrm{~mm}$, respectively. Besides, from the result, it has been found lower dual-frequency combinations even result in a better reconstruction performance when the lift-off of the sensor is over $14 \mathrm{~mm}$.

\section{ACKNOWLEDGEMENT}

This work was supported by [UK Engineering and Physical Sciences Research Council (EPSRC)] [grant number: EP/P027237/1] [title: Real-time In-line Microstructural Engineering (RIME)].

Author Contributions: (Methodology, manuscript drafting) M. Lu and X. Meng; (conceptualization, manuscript revision) M. Lu; (experiment data curation, manuscript review) M. Lu, R. Huang, L. Chen; (Supervision) W. Yin, A. Peyton, M. Lu. All authors have read and agreed to the published version of the manuscript.

\section{REFERENCES}

[1]. X. Chen and Y. Lei, "Electrical conductivity measurement of ferromagnetic metallic materials using pulsed eddy current method," NDT \& E International, vol. 75, pp. 33-38, 2015.

[2]. W. Li, X. Yuan, G. Chen, J. Ge, X. Yin and K. Li, "High sensitivity rotating alternating current field measurement for arbitrary-angle underwater cracks," NDT\&E International, vol. 79, pp. 123-131, Apr. 2016.

[3]. G. Yang, G. Dib, L. Udpa, A. Tamburrino and S. S. Udpa, "Rotating Field EC-GMR Sensor for Crack Detection at Fastener Site in Layered Structures," IEEE Sensors Journal, vol. 15, no. 1, pp. 463-470, Jan. 2015.

[4]. J. C. Moulder, E. Uzal, and J. H. Rose, "Thickness and conductivity of metallic layers from eddy current measurements," Review of Scientific Instruments, vol. 63, no. 6, 1992.

[5]. I. Z. Abidin, C. Mandache, G. Y. Tian and M. Morozov, "Pulsed eddy current testing with variable duty cycle on rivet joints," NDT \& E International, vol. 42, no. 7, pp. 599-605, 2009.

[6]. J. Kral et al., "The lift-off effect in eddy currents on thickness modeling and measurement," IEEE Transactions on Instrumentation and Measurement, vol. 62, no. 7, pp. 2043-2049, 2013.

[7]. E. Pinotti and E. Puppin, "Simple Lock-In Technique for Thickness Measurement of Metallic Plates," IEEE Transactions on Instrumentation and Measurement, vol. 63, no. 2, pp. 479-484, Feb. 2014.

[8]. A. L. Ribeiro et al., "Liftoff insensitive thickness measurement of aluminum plates using harmonic eddy current excitation and a GMR sensor," Measurement, vol. 45, no. 9, 2246-2253, 2012.

[9]. D. F. He et al., "Dual-frequency eddy-current NDE based on high-Tc rf SQUID," Physica C: Superconductivity, vol. 383, no. 3, pp. 223-226, 2002.

[10]. C. C. Tai, J. H. Rose, and J. C. Moulder, "Thickness and conductivity of metallic layers from pulsed eddy-current measurements," Review of Scientific Instruments, vol. 67, no. 11, Aug. 1996.

[11]. D. Vasic, V. Bilas and D. Ambrus, "Pulsed eddy-current nondestructive testing of ferromagnetic tubes," IEEE Transactions on Instrumentation and Measurement, vol. 53, no. 4, pp. 1289-1294, Aug. 2004.

[12]. M. Lu, et al. "Acceleration of frequency sweeping in eddy-current computation," IEEE Transactions on Magnetics, vol. 53, pp. 1-8, 2017.

[13]. Y. He, G. Tian, H. Zhang, M. Alamin, A. Simm and P. Jackson, "Steel Corrosion Characterization Using Pulsed Eddy Current Systems," IEEE Sensors Journal, vol. 12, no. 6, pp. 2113-2120, 2012.

[14]. G. Tytko and L. Dziczkowski, "E-Cored Coil With a Circular Air Gap Inside the Core Column Used in Eddy Current Testing," IEEE Transactions on Magnetics, vol. 51, no. 9, pp. 1-4, Sept. 2015.

[15]. T. Theodoulidis and E. E. Kriezis, "Eddy current canonical problems (with applications to nondestructive evaluation)", Material Science, 2006.

[16]. A. V. Egorov, V. V. Polyakov, D. S. Salita, E. A. Kolubaev, S. G. Psakhie, A. G. Chernyavskii, I. V. Vorobei, "Inspection of aluminum 
alloys by a multi-frequency eddy current method," Defence Technology, vol. 11, no. 2, pp. 99-103, 2015.

[17]. Y. Shin, D. Choi, Y. Kim and S. Lee, "Signal characteristics of differential-pulsed eddy current sensors in the evaluation of plate thickness," NDT \& E International, vol. 42, no. 3, pp. 215-221, 2009.

[18]. R. K. Amineh, et al, "Using AC field measurement data at an arbitrary lift-off distance to size long surface-breaking cracks in ferrous metals," NDT \& E Int., vol. 41, no. 3, pp. 169-177, 2008.

[19]. R. K. Amineh, et al, "Removal of probe lift-off effects on crack detection and sizing in metals by the AC field measurement technique," IEEE Trans. Magnetics, vol. 44, no. 8, pp. 2066-2073, 2008.

[20]. G. Y. Tian and A. Sophian, "Reduction of lift-off effects for pulsed eddy current NDT,” NDT \& E International, vol. 38, no. 4, pp. 319-324, 2005.

[21]. S. Giguere, B. A. Lepine, and J. M. S. Dubois. "Pulsed eddy current technology: Characterizing material loss with gap and lift-off variations," Journal of Research in Nondestructive Evaluation, vol. 13, no. 3, pp. 119129,2001

[22]. Y. Wang, M. Fan, B. Cao, B. Ye, and D. Wen, "Measurement of coating thickness using lift-off point of intersection features from pulsed eddy current signals," NDT \& E International, vol. 116, pp. 102333, 2020.

[23]. C.S. Angani et al. "Lift-off point of intersection feature in transient eddycurrent oscillations method to detect thickness variation in stainless steel," IEEE Transactions on Magnetics, vol. 52, no. 6, pp. 1-8, 2016.

[24]. D. Wen, M. Fan, B. Cao, B. Ye and G. Tian, "Extraction of LOI Features from Spectral Pulsed Eddy Current Signals for Evaluation of Ferromagnetic Samples," IEEE Sensors Journal, vol. 19, no. 1, pp. 189195, 1 Jan.1, 2019.

[25]. B. A. Abu-Nabah, "Reduction of lift-off effect in high-frequency apparent eddy current conductivity spectroscopy," Measurement Science and Technology, vol. 28, no.5, pp. 055107, 2017

[26]. W. Yin and A.J. Peyton, "Thickness measurement of non-magnetic plates using multi-frequency eddy current sensors," NDT \& E International, vol. 40, no. 1, pp. 43-48, 2007.

[27]. M. Lu, R. Huang, W. Yin, Q. Zhao, and A. Peyton, "Measurement of permeability for ferrous metallic plates using a novel lift-off compensation technique on phase signature," IEEE Sensors Journal, vol. 19, no. 17, pp. 7440-7446, 1 Sept.1, 2019.

[28]. M. Lu, et al. "Determination of the magnetic permeability, electrical conductivity, and thickness of ferrite metallic plates using a multifrequency electromagnetic sensing system," IEEE Transactions on Industrial Informatics, vol. 15, pp. 4111-4119, 2019.

[29]. J.R.S. Avila, M. Lu et al. "Accurate measurements of plate thickness with variable lift-off using a combined inductive and capacitive sensor," NDT \& E International, vol. 110, pp. 102202, 2020.

[30]. W. Yin, S. J. Dickinson, and A. Peyton, "Imaging the continuous conductivity profile within layered metal structures using inductance spectroscopy," IEEE Sensors Journal, vol. 5, no. 2, pp. 161-166, 2005.

[31]. M. Lu, H. Xu, W. Zhu, L. Yin et al. "Conductivity Lift-off Invariance and measurement of permeability for ferrite metallic plates," NDT \& E International, vol. 95, pp. 36-44, Apr. 2018.

[32]. M. Lu, L. Yin, A. J. Peyton and W. Yin, "A novel compensation algorithm for thickness measurement immune to lift-off variations using eddy current method," IEEE Transactions on Instrumentation and Measurement, vol. 65, no. 12, pp. 2773-2779, Dec. 2016.

[33]. M. Lu, X. Meng, W. Yin, Z. Qu, F. Wu, J. Tang, et al., "Thickness measurement of non-magnetic steel plates using a novel planar triple-coil sensor," NDT \& E International, vol. 107, 2019.

[34]. R. Huang, M. Lu, A. Peyton, and W. Yin, "Thickness measurement of metallic plates with finite planar dimension using eddy current method," IEEE Transactions on Instrumentation and Measurement, early access, 2020. Doi:10.1109/TIM.2020.2987413.

[35]. M. Lu et al. "Measurement of ferromagnetic slabs permeability based on a novel planar triple-coil sensor," IEEE Sensors J., vol. 20, no. 6, pp. 2904-2910, 2020.

[36]. M. Lu, W. Zhu, L. Yin, A. J. Peyton, W. Yin, and Z. Qu, "Reducing the lift-off effect on permeability measurement for magnetic plates from multifrequency induction data," IEEE Transactions on Instrumentation and Measurement, vol. 67, no. 1, pp. 167-174, Jan. 2018.
[37]. M. Fan et al., "Pulsed eddy current thickness measurement using phase features immune to liftoff effect," NDT \& E International, vol. 86, pp. 123-131, 2017

[38]. U. Burkhardt et al., "Mitigating the contrail cirrus climate impact by reducing aircraft soot number emissions," NPJ Climate and Atmospheric Science, vol. 1, no. 1, pp. 1-7, 2018.

[39]. T. Matsumoto et al., "Investigation of electromagnetic nondestructive evaluation of residual strain in low carbon steels using the eddy current magnetic signature (EC-MS) method," Journal of Magnetism and Magnetic Materials, vol. 479, pp. 212-221, 2019.

[40]. J. Tkocz et al., "High power phased EMAT arrays for nondestructive testing of as-cast steel," NDT \& E International, vol. 102, pp. 47-55, 2019.

[41]. C. V. Dodd, and W. E. Deeds, "Analytical solutions to eddy-current probe-coil problems," Journal of applied physics, vol. 39, no. 6, pp. 2829-2838, 1968.

[42]. W. Yin et al., "Analysis of the liftoff effect of phase spectra for eddy current sensors," IEEE Transactions on Instrumentation and Measurement, vol. 56, no. 6, pp. 2775-2781, 2007.

[43]. M. Lu et al., "Measuring lift-off distance and electromagnetic property of metal using dual-frequency linearity feature," IEEE Transactions on Instrumentation and Measurement, early access, 2020. DOI: 10.1109/TIM.2020.3029348.

[44]. M. Lu et al., "Thickness measurement of metallic film based on a highfrequency feature of triple-coil electromagnetic eddy current sensor," IEEE Transactions on Instrumentation and Measurement, early access, 2020. DOI: 10.1109/TIM.2020.3027929.

[45]. R. Huang et al., "Measuring Co-Axial Hole Size of Finite-Size Metallic Disk Based on a Dual-Constraint Integration Feature Using MultiFrequency Eddy Current Testing," IEEE Transactions on Instrumentation and Measurement, early access, 2020. DOI: 10.1109/TIM.2020.3026762

[46]. R. Huang et al., "Measurement of the radius of metallic plates based on a novel finite region eigenfunction expansion (FREE) method," IEEE Sensors Journal, early access, 2020. DOI: 10.1109/JSEN.2020.3009443

[47]. J. Tang et al., "A Novel Efficient FEM Thin Shell Model for BioImpedance Analysis," Biosensors, vol. 10, no. 6, pp. 69, 2020.

[48]. L. Chen, et al., "Textile Based Capacitive Sensor for Physical Rehabilitation via Surface Topological Modification," ACS Nano, vol. 14, no. 7, pp. 8191-8201, 2020. DOI: 10.1021/acsnano.0c01643

[49]. Z. Jin, et al., "Methods of Controlling Lift-off in Conductivity Invariance Phenomenon for Eddy Current Testing," IEEE ACCESS, vol. 8, pp. 2169-3536, 2020. DOI: 10.1109/ACCESS.2020.3007216.

[50]. J. Tang, et al., "Effect of frozen-thaw injury on cell membrane and bioimpedance," In 2020 IEEE International Instrumentation and Measurement Technology Conference (I2MTC), pp. 1-6. IEEE, 2020

[51]. J. Tang, et al., "Bio-impedance spectroscopy for frozen-thaw of biosamples: Non-contact inductive measurement and finite element (FE) based cell modelling," Journal of Food Engineering, vol. 272, pp. 109784, 2020.

[52]. R. Huang et al., "Methods of Controlling Lift-off in Conductivity Invariance Phenomenon for Eddy Current Testing," IEEE ACCESS, vol. 8, pp. 12438 - 12444, 2020. DOI: 10.1109/ACCESS.2020.2966032.

[53]. H. Xu et al., "Imaging a weld cross-section using a novel frequency feature in multi-frequency eddy current testing," Insight-NonDestructive Testing and Condition Monitoring, vol. 61, no. 12, pp. 738 743, 2019.

[54]. Y. Xie et al., "Novel Wearable Sensors for Biomechanical Movement Monitoring Based on Electromagnetic Sensing Techniques," IEEE Sensors Journal, vol. 20, no. 2, 2020. DOI: 10.1109/JSEN.2019.2943487

[55]. H. Xu et al., "Permeability invariance phenomenon and measurement of electrical conductivity for ferrite metallic plates," Insight-NonDestructive Testing and Condition Monitoring, vol. 61, no. 8, pp. 472 479, 2019.

[56]. M. Lu et al., "A model for the triboelectric nanogenerator with inductive load and its energy boost potential," Nano Energy, vol. 63, pp. 103883, 2019. 
[57]. W. Yin et al., "An equivalent-effect phenomenon in eddy current nondestructive testing of thin structures," IEEE ACCESS, vol. 7, pp. 70296 $-70307,2019$.

[58]. M. Lu et al., "Forward solver for deep earth exploration and induction logging using custom built Edge - Element FEM technique," Acta Geologica Sinica, vol. 93, pp. 302-304, 2019.

[59]. L. Chen et al., "Whole System Design of Wearable Magnetic Induction Sensor for Physical Rehabilitation," Advanced Intelligent Systems, vol. 1, no. 1, pp. 1900037, 2019.

[60]. Y. X et al., "A self-powered radio frequency (RF) transmission system based on the combination of triboelectric nanogenerator (TENG) and piezoelectric element for disaster rescue/relief," Nano Energy, vol. 54, pp. 331-340, 2018.

[61]. W. Yin et al., "Custom edge-element FEM solver and its application to eddy-current simulation of realistic $2 \mathrm{M}$-element human brain phantom," Bioelectromagnetics, vol. 39, no. 8, pp. 604-616, 2018.

[62]. L. Yin et al., "Detection of corrosion pits based on an analytically optimised eddy current sensor," Insight-Non-Destructive Testing and Condition Monitoring, vol. 60, no. 10, pp. 561-567, 2018.

[63]. W. Yin et al., "Acceleration of eddy current computation for scanning probes," Insight-Non-Destructive Testing and Condition Monitoring, vol. 60 , no. 10 , pp. 547-555, 2018.

[64]. W. Zhou et al., "Three-dimensional electromagnetic mixing models for dual-phase steel microstructures," Applied Sciences, vol. 8, no. 4, pp. 547-555, 2018.

[65]. M. Lu, et al., "Determining the magnetic permeability of ferrite steel strip by a custom inversion method," In Proc. 12th ECNDT, pp. 1-8. 2018.

[66]. J. Tang, et al., "Cellular structure analysis based on magnetic induction finite element method simulations and measurements," bioRxiv, pp. 275271, 2018. DOI: $10.1101 / 275271$

[67]. J.R.S. Avila, et al., "A novel dual modality sensor with sensitivities to permittivity, conductivity, and permeability," IEEE Sensors Journal, vol. 18, no. 1, pp. 356-362, 2017.

[68]. T. Yang, et al., "Level measurement for saline with a small surface area using high frequency electromagnetic sensing technique," Measurement, vol. 101, pp. 118-125, 2017.

[69]. M. Lu, et al., "Prediction of the asymptotical magnetic polarization tensors for cylindrical samples using the boundary element method," In 2015 IEEE Sensors Applications Symposium (SAS), pp. 1-4. IEEE, 2015.

[70]. M. Lu, et al., "Thickness Measurement of Metallic Film Based on a HighFrequency Feature of Triple-Coil Electromagnetic Eddy Current Sensor," Preprints, pp. 2020090717, 2020. DOI: 10.20944/preprints202009.0717.v1

[71]. R. Huang, et al., "Measuring Co-Axial Hole Size of Finite-Size Metallic Disk Based on a Dual-Constraint Integration Feature Using MultiFrequency Eddy Current Testing," Preprints, pp. 2020090601, 2020. DOI: 10.20944/preprints202009.0601.v1

[72]. Z. Jin, et al., "Methods of Controlling Lift-off in Conductivity Invariance Phenomenon for Eddy Current Testing," Preprints, pp. 2020050491, 2020. DOI: 10.20944/preprints202005.0491.v1 